


\title{
SOSTENIBILIDAD DEL SISTEMA SANITARIO EN EL ESTADO DEL BIENESTAR ${ }^{1}$
}

\author{
Autor: David Cantarero Prieto ${ }^{2}$ \\ Profesor Titular \\ Departamento de Economía \\ Responsable del Grupo de I+D en Economía de la Salud de la Universidad \\ de Cantabria
}

\section{Resumen}

En este artículo se enmarca al gasto sanitario dentro de los modernos Estados del Bienestar para describir la situación actual en cuanto a su financiación así como su problemática y retos de futuro a fin de poner en valor la aportación fundamental de la Economía de la Salud. Y es que, en un contexto de mejora de eficiencia y búsqueda de garantías de equidad, se trata de gastar mejor en sintonía con la realidad del sistema de financiación autonómica y coyuntura económica

\footnotetext{
${ }^{1} \mathrm{El}$ autor agradece las ayudas y comentarios previos de los revisores y secretario técnico de la Revista. En todo caso, cualquier error en este artículo corresponde a mi exclusiva responsabilidad.

${ }^{2}$ david.cantarero@unican.es
} 


\title{
David Cantarero Prieto
}

vigente en cada momento. Esto supone la necesidad de nuevos fondos y vincular el gasto a su coste-efectividad.

Palabras clave: gasto sanitario; financiación; Economía de la Salud; eficiencia; equidad.

\section{The value of Health economics after the Great Recession: expenditure and financing}

\begin{abstract}
This paper establishes the health expenditure within modern welfare states to describe the current situation in terms of funding as well as its problems and future challenges in order to value the fundamental contribution of Health Economics. And, in a framework of improving efficiency and seeking equity guarantees, the key is to spend better based on the regional financing system and economic situation. This implies the need for new funds and cost-effective tools for expenditure.
\end{abstract}

Key words: health expenditure; financing; Health Economics; efficiency; equity.

Recibido: 06/09/2016

Aceptado: 29/11/2016

\section{INTRODUCCIÓN}

Todo modelo que busque lo mejor en la gestión de sus servicios sanitarios busca conseguir las mayores cotas de calidad asistencial posible en contextos de disciplina presupuestaria como los actuales. Así, son los gestores del sistema los que tienen que utilizar de modo más eficiente y equitativo los recursos públicos escasos así como incorporar las mejores prácticas (benchmarking) en sanidad incluso procedentes de otros sectores (transparencia, incentivos a los proveedores, uso de sistemas de información, mejora de la productividad, etc.). En síntesis, siendo primordial controlar el gasto no olvidemos que la sostenibilidad del sistema y su buen funcionamiento obedece a la buena labor de nuestros profesionales sanitarios y ahí es fundamental el papel de los sistemas sanitarios públicos (Hansen et al., 2015; Cantarero y Oliva, 2016). Igualmente, el conocimiento aplicable a la práctica diaria del decisor sanitario ha de tener como guía fundamental a la mejora de la eficiencia y reducción de desigualdades (Pascual y Cantarero, 2013). 
Por consiguiente, los principales objetivos del presente artículo se basan en conocer la tendencia actual de los niveles de gasto y financiación sanitaria así como su comparativa correspondiente en el caso de nuestro país y su estructura descentralizada. Para ello, la hipótesis con la que se inicia el trabajo es pretender contrastar si el sistema español aún sigue la estela de otros países en cuanto a grado de recursos que gestiona el sector sanitario y ello viene condicionado por un sistema de financiación sin un alto grado de corresponsabilidad fiscal (entendida como una mayor autonomía y grado de respuesta sobre los tributos subcentrales y su capacidad de financiar los servicios públicos autonómicos). La metodología que se va a seguir para verificar dicha hipótesis será analizar las correspondientes series y últimas estadísticas al respecto tanto a nivel de los principales países desarrollados del mundo como del Ministerio de Hacienda y Administraciones Públicas para el caso de nuestro país.

Explicadas así las principales líneas de desarrollo y valor de la problemática actual en sanidad a continuación, se describen las principales secciones del artículo. En el apartado segundo pasamos a enmarcar al gasto sanitario dentro del gasto social de los modernos Estados de Bienestar. En la sección tercera se incluye una breve descripción de la financiación sanitaria así como su problemática actual y retos de futuro a fin de poner en valor la aportación de la ·Economía de la Salud. El último apartado del presente trabajo incluye las conclusiones y principales recomendaciones.

\section{EL GASTO SANITARIO Y SU INCLUSIÓN DENTRO DEL ESTADO DEL BIENESTAR}

Los sistemas sanitarios de provisión y financiación en comparación con los de otro tipo de bienes tienen históricamente un alto grado de intervención pública. No obstante, la gran mayoría de los bienes y servicios que constituyen dicho "gasto" (por ejemplo, la sanidad) son realmente bienes de tipo privado, ya que en ellos su consumo es rival (sus beneficios en cuanto a consumo pueden dividirse entre los agentes implicados) y se les aplica el principio de exclusión (técnicamente se les puede aplicar un precio unitario, excepto a servicios como la investigación o Salud pública). Ambas características hacen que la sanidad pueda ser comercializable e incluso producida privadamente aunque, por diversos motivos, en la mayoría de países desarrollados de la OCDE (Organización para la Cooperación y Desarrollo Económico) se financia y produce principalmente por el sector público. En todo caso, en la sanidad, y a diferencia de otros servicios, realmente tanto el progreso tecnológico como las innovaciones terapéuticas incrementan significativamente las opciones de tratamiento.

En ese sentido, según la OECD Health Data y OECD Health at a Glance (2016), la evolución actual demográfica muestra una clara tendencia al envejecimiento 


\section{David Cantarero Prieto}

estimándose un consumo en servicios sanitarios de los mayores de 65 años muy superior al resto. Así, la asistencia sanitaria es uno de los sectores económicos más importantes en los 34 países de la OCDE (Tablas 1 y 2) y es que en porcentaje de gasto sanitario sobre el PIB, Estados Unidos sigue siendo en 2015 el país del mundo con mayor gasto en sanidad (16,9\%, del PIB en 2015) mientras que Noruega lo es en términos per cápita (5598 dólares en Paridades de Poder de Compra). A este respecto, cabe indicar que en la mayoría de países el sector público tiene un papel primordial en la financiación al suponer en media en el año 2015 un $72,9 \%$ del total de gasto sanitario para media de la OCDE. Sólo son excepción en cuanto a esa tendencia países como Estados Unidos, México y Corea donde el sector privado tiene un papel central. De esta manera, España junto con Australia, Canadá, Noruega y Portugal se basan fundamentalmente en sistemas sanitarios financiados con impuestos bien centrales bien compartidos por los diferentes niveles de gobierno con bajo peso relativo de fondos de aseguramiento frente a los casos de Alemania, Países Bajos, Polonia, Francia y Japón.

Tabla 1. Gasto sanitario (público y privado) en porcentaje del PIB

\begin{tabular}{|l|r|r|r|r|}
\hline \multicolumn{1}{|c|}{ Países / años } & \multicolumn{2}{|c|}{2012} & 2014 & \multicolumn{2}{c|}{2015} \\
\hline Australia & 8,5 & 8,7 & 9,0 & 9,3 \\
\hline Austria & 10,1 & 10,1 & 10,3 & 10,3 \\
\hline Bélgica & 9,9 & 10,2 & 10,4 & 10,4 \\
\hline Canadá & 10,7 & 10,3 & 10,0 & 10,2 \\
\hline Chile & 6,7 & 7,0 & 7,7 & 7,8 \\
\hline República Checa & 6,9 & 7,1 & 7,7 & 7,6 \\
\hline Dinamarca & 10,4 & 10,3 & 10,6 & 10,6 \\
\hline Estonia & 6,3 & 5,8 & 6,1 & 6,3 \\
\hline Finlandia & 8,9 & 9,3 & 9,5 & 9,6 \\
\hline Francia & 10,7 & 10,8 & 11,1 & 11,0 \\
\hline Alemania & 11,0 & 10,8 & 11,0 & 11,1 \\
\hline Grecia & 9,9 & 8,9 & 8,3 & 8,2 \\
\hline Hungría & 7,6 & 7,5 & 7,2 & 7,0 \\
\hline Islandia & 8,8 & 8,7 & 8,9 & 8,8 \\
\hline Irlanda & 10,6 & 10,1 & 10,1 & 9,4 \\
\hline Israel & 7,0 & 7,1 & 7,6 & 7,4 \\
\hline Italia & 9,0 & 8,8 & 9,1 & 9,1 \\
\hline Japón & 9,5 & 11,2 & 11,4 & 11,2 \\
\hline
\end{tabular}




\begin{tabular}{|l|r|r|r|r|}
\hline \multicolumn{1}{|c|}{ Países / años } & \multicolumn{2}{|c|}{2010} & \multicolumn{1}{c|}{2014} & \multicolumn{1}{c|}{2015} \\
\hline Corea & 6,4 & 6,6 & 7,1 & 7,2 \\
\hline Letonia & 6,2 & 5,4 & 5,5 & 5,6 \\
\hline Luxemburgo & 7,1 & 6,7 & 6,3 & 7,2 \\
\hline México & 6,2 & 6,0 & 5,8 & 5,9 \\
\hline Países Bajos & 10,4 & 10,9 & 10,9 & 10,8 \\
\hline Nueva Zelanda & 9,7 & 9,7 & 9,4 & 9,4 \\
\hline Noruega & 8,9 & 8,8 & 9,3 & 9,9 \\
\hline Polonia & 6,4 & 6,2 & 6,4 & 6,3 \\
\hline Portugal & 9,8 & 9,3 & 9,0 & 8,9 \\
\hline Eslovaquia & 7,8 & 7,7 & 7,0 & 7,0 \\
\hline Eslovenia & 8,6 & 8,7 & 8,5 & 8,4 \\
\hline España & 9,0 & 9,1 & 9,1 & 9,0 \\
\hline Suecia & 8,5 & 10,9 & 11,2 & 11,1 \\
\hline Suiza & 10,5 & 11,0 & 11,4 & 11,5 \\
\hline Turquía & 5,3 & 5,0 & 5,1 & 5,2 \\
\hline Reino Unido & 8,5 & 8,5 & 9,9 & 9,8 \\
\hline Estados Unidos & 16,4 & 16,4 & 16,6 & 16,9 \\
\hline Media de la OCDE-34 países & 9,1 & 9,1 & 9,2 & 9,3 \\
\hline
\end{tabular}

Fuente: OECD Health Data (2016).

\section{Tabla 2. Gasto sanitario per cápita (público y privado)} en Paridades de Poder de Compra (dólares)

\begin{tabular}{|l|r|r|r|r|}
\hline \multicolumn{1}{|c|}{ Países / años } & \multicolumn{1}{c|}{2010} & \multicolumn{1}{c|}{2012} & \multicolumn{1}{c|}{2014} & \multicolumn{1}{c|}{2015} \\
\hline Australia & 2476 & 2572 & 2804 & 2950 \\
\hline Austria & 3226 & 3528 & 3715 & 3800 \\
\hline Bélgica & 3015 & 3324 & 3508 & 3576 \\
\hline Canadá & 3008 & 3051 & 3178 & 3266 \\
\hline Chile & 555 & 687 & 1022 & 1069 \\
\hline República Checa & 1566 & 1699 & 1993 & 2069 \\
\hline Dinamarca & 3696 & 3874 & 4089 & 4160 \\
\hline Estonia & 1018 & 1152 & 1304 & 1378 \\
\hline Finlandia & 2541 & 2847 & 2918 & 3009 \\
\hline Francia & 3015 & 3176 & 3435 & 3473 \\
\hline
\end{tabular}


David Cantarero Prieto

\begin{tabular}{|l|r|r|r|r|}
\hline \multicolumn{1}{|c|}{ Países / años } & \multicolumn{1}{|c|}{2010} & \multicolumn{1}{c|}{2012} & \multicolumn{1}{c|}{2015} \\
\hline Alemania & 3650 & 3921 & 4332 & 4477 \\
\hline Grecia & 1998 & 1550 & 1324 & 1360 \\
\hline Hungría & 1096 & 1117 & 1205 & 1236 \\
\hline Islandia & 2739 & 2825 & 3158 & 3283 \\
\hline Irlanda & 3493 & 3533 & 3466 & 3550 \\
\hline Israel & 1274 & 1419 & 1567 & 1582 \\
\hline Italia & 2438 & 2445 & 2431 & 2470 \\
\hline Japón & 2626 & 3371 & 3512 & 3523 \\
\hline Corea & 1153 & 1216 & 1334 & 1384 \\
\hline Letonia & 645 & 693 & 775 & 828 \\
\hline Luxemburgo & 3720 & 3650 & 3745 & 4329 \\
\hline México & 471 & 537 & 554 & 559 \\
\hline Países Bajos & 4066 & 4128 & 4254 & 4312 \\
\hline Nueva Zelanda & 2434 & 2563 & 2817 & 2862 \\
\hline Noruega & 4438 & 4864 & 5176 & 5598 \\
\hline Polonia & 961 & 1025 & 1162 & 1201 \\
\hline Portugal & 1846 & 1663 & 1711 & 1737 \\
\hline Republica de Eslovaquia & 1379 & 1443 & 1581 & 1663 \\
\hline Eslovenia & 1733 & 1785 & 1846 & 1910 \\
\hline España & 2181 & 2107 & 2131 & 2204 \\
\hline Suecia & 2902 & 4062 & 4223 & 4375 \\
\hline Suiza & 3444 & 4069 & 4423 & 4711 \\
\hline Turquía & 721 & 768 & 821 \\
\hline Reino Unido & 2610 & 3160 & 3171 \\
\hline Estados Unidos & 4063 & 4448 & 4672 \\
\hline Media de la OCDE-34 países & 2567 & 2737 & 2840 \\
\hline
\end{tabular}

Fuente: OECD Health Data (2016).

La comparativa internacional que muestran las Tablas 1 y 2 deshace ciertas equivocaciones en cuanto a la cuantificación del gasto pues se observa que no “más” es siempre mejor. Sólo así se entiende que Noruega tenga en el año 2015 más gasto sanitario en relación al PIB (9,9\%) que España (9\%), debido a los recortes sanitarios sufridos en estos últimos años y nuestro menor PIB relativo, aunque tengamos mejores indicadores de salud que los noruegos, por ejemplo, en esperanza de vida al nacer. 
Además, respecto al contexto europeo, nuestro modelo sanitario no difiere mucho del de aquellos países que pueden considerarse precursores de los sistemas sanitarios actuales. Así, dejando a un lado sistemas privados como el de Estados Unidos o mixtos como el de otros países de la OCDE, en la Unión Europea (UE) todos los países disponen de uno de estos dos sistemas:

a) Sistemas de Seguro Sanitario Social (SS), o de corte bismarkiano (inspirados en la legislación social alemana de 1883) o sistemas profesionales en los que existe una afiliación obligatoria general donde el Estado especifica las prestaciones incluidas y el suministro de servicios se reserva a fondos de aseguramiento con autorregulación por parte de los proveedores. Se financian por cotizaciones de empresarios y empleados y la propiedad de los medios de producción, así como la provisión de los cuidados de salud, es pública y privada. Este modelo lo siguen países como Austria, Bélgica, Francia, Alemania, Holanda y Luxemburgo.

b) Sistemas Nacionales de Salud (SNS), que toman como base el Informe Beveridge de 1942, cuyo fundamento es un acceso y cobertura casi universal (libre acceso de todos los ciudadanos), con financiación impositiva (y esquemas de pago directo por los pacientes, en general, de escasa cuantía), una provisión directamente regulada y profesionales (asalariados o retribuidos a partir de fórmulas de tipo capitativo) empleados por el Estado, reconociendo para el sector privado un papel complementario o residual. Se incluyen dentro de este sistema a países como Dinamarca, Finlandia, Irlanda, Suecia y Reino Unido después de la II Guerra Mundial, mientras que Grecia, Italia, Portugal y España se unieron al SNS en los ochenta.

Precisamente, en lo que se refiere al caso de España, si nos detenemos por un momento en los gastos de tipo social en el período 2007-2013 a partir del correspondiente desglose de gasto por funciones hemos de tener en cuenta que el año base del sistema de financiación fue 2007 en un contexto donde estaban finalizadas las principales transferencias a las distintas Comunidades Autónomas (CCAA).

Así, según puede observarse en el Gráfico 1 a partir de datos de la IGAE (Intervención General de la Administración del Estado) y Eurostat según el Sistema Europeo de Cuentas-SEC (y no teniendo en cuenta el gasto de transferencias entre Administraciones Públicas) el mayor volumen de gasto social se alcanzó en el año 2009 (11,5\% del PIB) habiéndose reducido considerablemente a partir de dicho momento por los recortes realizados para la reducción del déficit, si bien en 2013 se situaba en el 10,4\% del PIB superior al 9,6\% registrado en 2007 (año base del sistema de financiación autonómica). Y es que la Gran Recesión en España tuvo como consecuencia principal desde 2007 elevados niveles de desempleo y 


\section{David Cantarero Prieto}

una crisis fiscal que aún hoy sitúa nuestros niveles de deuda pública que ya han superado el 100\% del PIB y ha lastrado el crecimiento de muchos gastos públicos y sociales y, por supuesto, también del de tipo sanitario.

\section{Gráfico 1. Evolución de los gastos autonómicos de tipo social en porcentaje del PIB (2007-2013)}

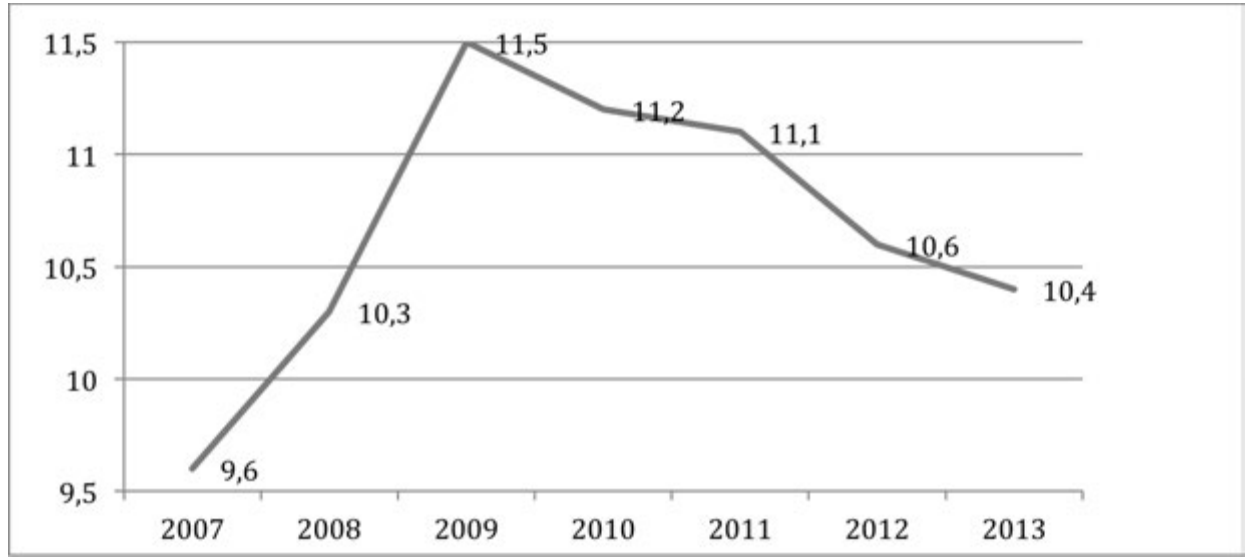

Fuente: elaborado a partir de datos de la IGAE y Eurostat según el Sistema Europeo de Cuentas-SEC.

En las Tablas 3 y 5 se muestra la asignación territorial del gasto para cada autonomía en el año base del modelo de financiación (2007), el de su aplicación efectiva (2009) y el último liquidado (2013). Así, igualmente que en el caso de la educación, el gasto público sanitario per cápita presenta una evolución decreciente si comparamos el año 2013 con 2007 mostrando así como en 2013 las CC. AA. con mayores niveles de gasto son Asturias, Cantabria, Galicia, La Rioja, Murcia, Aragón, Extremadura y Castila y León mientras que Andalucía o Illes Balears presentan los niveles más bajos. En todo caso, a la vista de los correspondientes índices de dispersión, las diferencias en gasto per cápita en sanidad entre CC. AA. son menores que en educación, tal y como también se ha reflejado recientemente en otros trabajos (Conde Ruiz et al., 2016; Fundación BBVA e IVIE, 2015).

Por tanto, a la vista de situaciones en las que hay CC. AA. ricas que gastan menos que la media y otras relativamente mucho más pobres en las que se gasta más no es únicamente el nivel de renta de la CC. AA. el causante principal de las diferencias. Resultan pues relevantes otros factores como, entre otros, la propia configuración del modelo de financiación autonómica o el peso económico del gasto que originan pacientes con enfermedades crónicas (como cardiovasculares, 
diabetes, cáncer, dolencias psiquiátricas, pulmonares, etc.) y/o de avanzada edad que residen en cada CC. AA. Además, la mitad de la población sufre al menos una enfermedad crónica mientras que una vez pasados los 65 años, tienen de media cuatro enfermedades de ese tipo (López-Casasnovas y López-Valcarcel, 2016).

El Gráfico 2 muestra cómo el gasto per cápita en sanidad ha experimentado una disminución en todas la CC. AA. en el año 2013 frente al 2009 alcanzándose valores incluso inferiores al correspondiente dato en 2007. Asimismo, al principio del periodo considerado, en el año 2007, la dispersión del gasto sanitario era mayor (coeficiente de variación del 0,11) que en el año 2013 (coeficiente de variación del 0,08).

Tabla 3. Indicadores de gasto social por Comunidades Autónomas año 2007

\begin{tabular}{|l|r|r|r|r|}
\hline & \multicolumn{2}{|c|}{ Educación (a) } & \multicolumn{2}{c|}{ Sanidad (b) } \\
\hline Concepto & Gasto per cápita & Índice base $=100$ & Gasto per cápita & Índice base $=100$ \\
\hline Cataluña & 1102,26 & 105,32 & 1523,96 & 95,62 \\
\hline Galicia & 1052,41 & 100,56 & 1526,88 & 95,80 \\
\hline Andalucía & 1033,62 & 98,76 & 1349,29 & 84,66 \\
\hline Asturias & 1054,22 & 100,73 & 1645,23 & 103,23 \\
\hline Cantabria & 1086,03 & 103,77 & 1817,80 & 114,05 \\
\hline La Rioja & 998,30 & 95,39 & 2130,06 & 133,65 \\
\hline Murcia & 1098,03 & 104,92 & 1533,99 & 96,25 \\
\hline C. Valenciana & 1047,66 & 100,10 & 1422,79 & 89,27 \\
\hline Aragón & 1015,13 & 96,99 & 1636,82 & 102,70 \\
\hline C. La Mancha & 1081,50 & 103,34 & 1551,29 & 97,33 \\
\hline Canarias & 1061,02 & 101,38 & 1596,40 & 100,16 \\
\hline Extremadura & 1087,43 & 103,90 & 1724,41 & 108,19 \\
\hline Baleares & 928,64 & 88,73 & 1472,74 & 92,40 \\
\hline Madrid & 1003,07 & 95,84 & 1468,62 & 92,15 \\
\hline C. León & 1049,38 & 100,27 & 1506,90 & 94,55 \\
\hline Promedio & 1046,58 & 100,00 & 1593,81 & 100,00 \\
\hline $\begin{array}{l}\text { Coeficiente } \\
\text { de Variación }\end{array}$ & 0,04 & & 0,11 & \\
\hline
\end{tabular}

Nota: los gastos per cápita están considerados en términos constantes de 2013.

Fuente: elaborado a partir de datos de la IGAE, Ministerio de Hacienda y Administraciones Públicas, Ministerio de Sanidad, Servicios Sociales e Igualdad, Ministerio de Educación, Cultura y Deporte y BBVA e IVIE. 
Tabla 4. Indicadores de gasto social por Comunidades Autónomas año 2009

\begin{tabular}{|l|r|r|r|r|}
\hline & \multicolumn{2}{|c|}{ Educación (a) } & \multicolumn{2}{c|}{ Sanidad (b) } \\
\hline Concepto & Gasto per cápita & Índice base $=100$ & Gasto per cápita & Índice base $=100$ \\
\hline Cataluña & 1203,40 & 105,86 & 1726,54 & 98,06 \\
\hline Galicia & 1142,65 & 100,52 & 1738,49 & 98,74 \\
\hline Andalucía & 1152,99 & 101,43 & 1514,22 & 86,00 \\
\hline Asturias & 1041,80 & 91,65 & 1964,42 & 111,57 \\
\hline Cantabria & 1177,81 & 103,61 & 1711,55 & 97,21 \\
\hline La Rioja & 1066,29 & 93,80 & 1770,23 & 100,54 \\
\hline Murcia & 1252,50 & 110,18 & 1886,22 & 107,13 \\
\hline C. Valenciana & 1199,10 & 105,48 & 1629,26 & 92,53 \\
\hline Aragón & 1122,11 & 98,71 & 1866,47 & 106,01 \\
\hline C. La Mancha & 1168,64 & 102,81 & 1882,45 & 106,91 \\
\hline Canarias & 1109,41 & 97,59 & 1826,87 & 103,76 \\
\hline Extremadura & 1199,40 & 105,51 & 1944,82 & 110,46 \\
\hline Baleares & 1045,72 & 91,99 & 1576,85 & 89,56 \\
\hline Madrid & 1044,32 & 91,87 & 1667,75 & 94,72 \\
\hline C. León & 1125,15 & 98,98 & 1704,33 & 96,80 \\
\hline Promedio & 1136,75 & 100,00 & 1760,70 & 100,00 \\
\hline $\begin{array}{l}\text { Coeficiente } \\
\text { de Variación }\end{array}$ & 0,05 & & 0,07 & \\
\hline
\end{tabular}

Nota: los gastos per cápita están considerados en términos constantes de 2013.

Fuente: elaborado a partir de datos de la IGAE, Ministerio de Hacienda y Administraciones Públicas, Ministerio de Sanidad, Servicios Sociales e Igualdad, Ministerio de Educación, Cultura y Deporte y BBVA e IVIE.

Tabla 5. Indicadores de gasto social por Comunidades Autónomas año 2013

\begin{tabular}{|l|r|r|r|r|}
\hline & \multicolumn{2}{|c|}{ Educación (a) } & \multicolumn{2}{c|}{ Sanidad (b) } \\
\hline Concepto & Gasto per cápita & Índice base $=100$ & Gasto per cápita & Índice base = 100 \\
\hline Cataluña & 890,00 & 96,84 & 1322,74 & 94,08 \\
\hline Galicia & 914,89 & 99,55 & 1429,88 & 101,70 \\
\hline Andalucía & 959,35 & 104,39 & 1182,17 & 84,08 \\
\hline Asturias & 1066,56 & 116,05 & 1714,10 & 121,91 \\
\hline Cantabria & 1033,07 & 112,41 & 1530,48 & 108,85 \\
\hline La Rioja & 890,33 & 96,88 & 1429,49 & 101,67 \\
\hline Murcia & 994,83 & 108,25 & 1453,39 & 103,37 \\
\hline C. Valenciana & 866,74 & 94,31 & 1301,09 & 92,54 \\
\hline Aragón & 906,74 & 98,66 & 1487,38 & 105,79 \\
\hline C. La Mancha & 858,79 & 93,44 & 1334,25 & 94,90 \\
\hline
\end{tabular}




\begin{tabular}{|l|r|r|r|r|}
\hline & \multicolumn{2}{|c|}{ Educación (a) } & \multicolumn{2}{|c|}{ Sanidad (b) } \\
\hline Canarias & 846,10 & 92,06 & 1332,20 & 94,75 \\
\hline Extremadura & 1007,75 & 109,65 & 1463,85 & 104,12 \\
\hline Baleares & 830,76 & 90,39 & 1274,53 & 90,65 \\
\hline Madrid & 790,73 & 86,04 & 1377,19 & 97,95 \\
\hline C. León & 928,90 & 101,07 & 1457,13 & 103,64 \\
\hline Promedio & 919,04 & 100,00 & 1405,99 & 100,00 \\
\hline $\begin{array}{l}\text { Coeficiente } \\
\text { de Variación }\end{array}$ & 0,08 & & 0,08 & \\
\hline
\end{tabular}

Nota: los gastos per cápita están considerados en términos constantes de 2013.

Fuente: elaborado a partir de datos de la IGAE, Ministerio de Hacienda y Administraciones Públicas, Ministerio de Sanidad, Servicios Sociales e Igualdad, Ministerio de Educación, Cultura y Deporte y BBVA e IVIE.

\section{Gráfico 2. Evolución del gasto per cápita: Sanidad (2007, 2009 y 2013)}

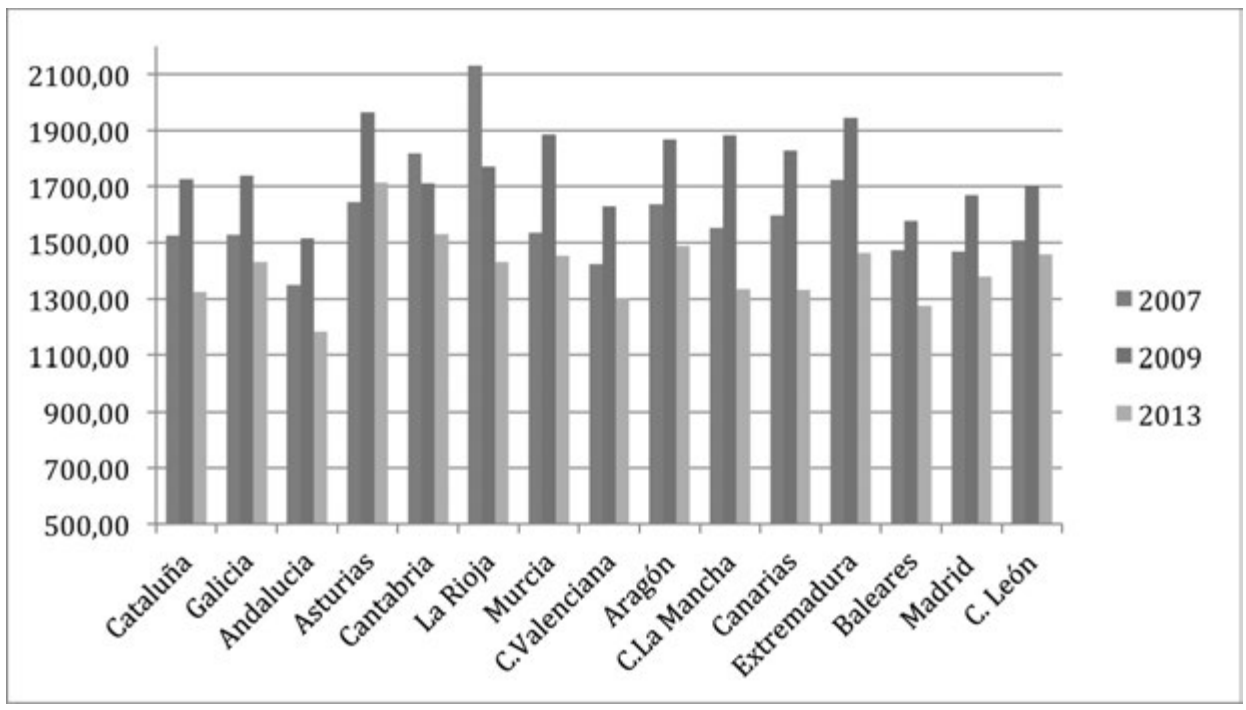

Fuente: elaborado a partir de datos de la IGAE, Ministerio de Hacienda y Administraciones Públicas, Ministerio de Sanidad, Servicios Sociales e Igualdad, Ministerio de Educación, Cultura y Deporte y BBVA e IVIE.

Volviendo a la comparativa entre países, cabe señalar que el grado de descentralización está ligado a determinantes de tipo político y al modelo empleado ("privado" como en Estados Unidos o público bien de SS o SNS) en cada caso, siendo mayor en los modelos privados, al descentralizar éstos gran parte de las 


\section{David Cantarero Prieto}

responsabilidades a los propios usuarios. Por ello, una de las mejores explicaciones de este importante papel del sector público en sanidad descansa en la adopción de un análisis específico del sector desde una perspectiva puramente normativa (Economía del Bienestar). Es decir, se debería fundamentar en la adopción de juicios de valor (teóricos) que suponen un "campo de batalla” ideológico y social.

Por eso, la intervención estatal pretende aumentar el bienestar social cuando no se cumplían alguna de las hipótesis que optimizan la asignación de recursos del "libre mercado" (oferta y demanda). Igualmente, a través de la intervención pública se evitan monopolios u oligopolios (concentración del poder de mercado en sólo uno o pocos oferentes para influir en los precios) que podrían generar nuevas inequidades (por ejemplo, en el acceso a terapias innovadoras). En síntesis, tal y como afirman López-Casasnovas (2015) y López-Casasnovas y López-Valcarcel (2016), hay que abandonar esa idea permanente de que "todo va mal" y poner en valor lo conseguido para seguir así modernizando el Estado del Bienestar a los tiempos actuales.

En este contexto la sanidad es uno de los pilares fundamentales de nuestro sistema y el derecho a la protección de la salud aparece recogido en la Constitución Española de 1978 habiéndose a su vez consolidado junto con el cambio desde un modelo de SS al actual SNS (desde la Ley General de Sanidad de 1986) descentralizado desde el año 2002 (excepto en Ceuta y Melilla). Asimismo, la Ley 16/2003, de 28 de Mayo, de Cohesión y Calidad del SNS, sigue siendo el marco legal para garantizar la coordinación y cooperación de los distintos Servicios Regionales de Salud. A su vez, la Ley 33/2011, de 4 de octubre, General de Salud Pública, marcó las directrices en cuanto a prevención que luego fueron modificadas por el Real Decreto Ley 16/2012, de 20 de abril, de medidas urgentes para garantizar la sostenibilidad del Sistema Nacional de Salud y mejorar la calidad y seguridad de sus prestaciones en un contexto de crisis y recortes presupuestarios.

Por tanto, el dilema de cómo ajustar la financiación al gasto en sanidad no es sencillo de resolver pues en ese sector la oferta (dónde el médico tiene una labor fundamental y la innovación tecnológica es una de las mayores fuentes de aumento del gasto) es la que suele condicionar la demanda así como la protección del sistema público (y, por exclusión, que quedaría a merced de posibles copagos "evitables o no"). Y es que los problemas presupuestarios como resultado de un importante desfase entre el crecimiento de los ingresos y gastos autonómicos afectan de manera importante a la sanidad y su elevado nivel de endeudamiento actual.

\section{FINANCIACIÓN, RETOS DE FUTURO Y VALOR DE LA APORTA- CIÓN EN SANIDAD}

Tras sucesivas reformas en el sistema de financiación sanitaria primero en su modo finalista de entenderlo y luego desde hace ya catorce años desde una 
perspectiva general, pasamos a analizar cómo se sufragan las necesidades globales de financiación. En especial, vamos a fijar nuestra atención en las referentes al gasto sanitario dado que los recursos del modelo se componen de la capacidad tributaria propia de las CC. AA. y las transferencias de recursos procedentes tanto del Fondo de Garantía de los Servicios Públicos Fundamentales (FGSPF), como del Fondo de Suficiencia Global. Además, las distintas CC. AA. reciben los recursos que les corresponde por su participación en los Fondos de Convergencia (Mones et al., 2016). De este modo, según las últimas liquidaciones disponibles del Ministerio de Hacienda y Administraciones Públicas puede observarse cierta mejora en la capacidad financiera de las CC. AA. aunque de modo desigual.

En este punto, resulta primordial tener en cuenta el papel que desempeña la Transferencia del FGSPF cuyo objetivo se basa en pretender asegurar que cada CC. AA. reciba los mismos recursos por habitante ajustado para financiar los Servicios Públicos Fundamentales vinculados al Estado del Bienestar. Tal y como se establece en el modelo actual, dicha transferencia de garantía para cada CC. AA. será el saldo, positivo o negativo, que resulte de la diferencia entre la participación que le corresponda y el 75\% de sus recursos tributarios normativos.

De este modo, la participación de cada CC. AA. en dicho FGSPF ha venido basándose en un indicador sintético, denominado población ajustada, compuesto por las siguientes variables: población, con una ponderación del 30\%; superficie, con una ponderación del 1,8\%; dispersión, con una ponderación del 0,6\%, en función del número de entidades singulares, núcleos de población de cada CC. AA.; insularidad, con una ponderación de 0,6\%, imputada proporcionalmente a la distancia en kilómetros, ponderada por tramos, entre las costas de la península y las capitales insulares; población protegida equivalente, con una ponderación del 38\%, distribuida en siete grupos de edad con arreglo a la metodología del Informe del Grupo de Trabajo de Análisis del Gasto Sanitario con la aplicación de ponderaciones a la población protegida (niños entre 0 a 4 años: 1,031; entre 5 y 14 años: 0,433; entre 15 y 44 años: 0,547; entre 45 y 54 años: 0,904; entre 55 y 64 años: 1,292; entre 65 y 74 años: 2,175; a partir de 75 años: 2,759); población mayor de 65 años, con una ponderación del 8,5\%; población entre 0 y 16 años, con una ponderación del 20,5\%.

De hecho, es importante tener en cuenta que aunque el modelo incorporó nuevas variables (para determinar las necesidades de gasto sanitario y educativo así como un ajuste anual de las mismas) pensamos firmemente que se puede avanzar más investigando el efecto que otros parámetros puedan tener tanto sobre el montante total de financiación como su reparto.

Así, el número de personas dependientes o de aquellas que padecen enfermedades crónicas podrían servir para aproximar mejor el gasto real de tipo social de las CC. AA.. Por consiguiente, han de validarse nuevas variables de necesidades de gasto a fin de reflejar mejor el margen de maniobra con el que las CC. AA. 


\section{David Cantarero Prieto}

pueden contar en términos de suficiencia financiera. Y es que la falta de actualización periódica de los recursos destinados a las CC. AA. según la evolución de sus necesidades de gasto viene siendo una de las principales justificaciones para que cualquier CC. AA. solicite periódicamente la revisión del sistema de financiación.

Por último, la Tabla 6 recoge la liquidación del sistema de financiación autonómica correspondiente al último ejercicio (2013) recogido a fecha de cierre de este artículo en un contexto general de descentralización asimétrica. En ese sentido, puede observarse cómo es el modelo de financiación autonómica el que cubre gran parte del gasto social relativo a sanidad y educación (102,99\% de cobertura en 2013). A pesar de todo lo anterior, siguen necesitándose otras fuentes financieras en especial en aquellas CC. AA. que sostienen en los últimos tiempos que precisan de cambios sustanciales en el nuevo diseño del modelo de financiación autonómica.

\section{Tabla 6. Liquidación definitiva del sistema de financiación autonómica en el año 2013 (miles de euros)}

\begin{tabular}{|c|c|c|c|c|c|c|c|c|c|}
\hline $\begin{array}{l}\text { Comuni- } \\
\text { dades } \\
\text { Autóno- } \\
\text { mas }\end{array}$ & $\begin{array}{l}\text { Capacidad } \\
\text { Tributaria }\end{array}$ & $\begin{array}{l}\text { Transfe- } \\
\text { rencia } \\
\text { del Fondo } \\
\text { de } \\
\text { Garantía }\end{array}$ & \begin{tabular}{|c|} 
Partici- \\
pación en \\
el Fondo \\
Suficiencia \\
Global \\
\end{tabular} & $\begin{array}{c}\text { Total } \\
\text { Fondos } \\
\text { Conver- } \\
\text { gencia }\end{array}$ & $\begin{array}{l}\text { Finan- } \\
\text { ciación } \\
\text { normativa } \\
\text { total }\end{array}$ & $\begin{array}{l}\text { Competen } \\
\text { Específicas }\end{array}$ & $\begin{array}{c}\text { Financia- } \\
\text { ción } \\
\text { total } \\
\text { homogénea }\end{array}$ & $\begin{array}{l}\text { Recursos } \\
\text { totales del } \\
\text { sistema de } \\
\text { financia- } \\
\text { ción }\end{array}$ & $\begin{array}{l}\text { \% cobertu- } \\
\text { ra del } \\
\text { sistema de } \\
\text { sanidad y } \\
\text { educación } \\
\end{array}$ \\
\hline Cataluña & 17361542 & -839309 & 734578 & 684358 & 17941169 & 2267389 & 14989421 & 17256811 & 96,86 \\
\hline Galicia & 5038950 & 1097783 & 537321 & 262336 & 6936390 & 292019 & 6382034,74 & 6674054 & 97,17 \\
\hline Andalucía & 13125531 & 3546077 & 494021 & 420012 & 17585643 & 785806 & 16379824 & 17165630 & 105,30 \\
\hline Asturias & 2227062 & 184131 & 169879 & 100217 & 2681290 & 72767 & 2508306 & 2581073 & 115,08 \\
\hline Cantabria & 1323281 & -13187 & 424053 & 54498 & 1788646 & 124739 & 1609407 & 1734147 & 87,50 \\
\hline La Rioja & 656853 & 51008 & 184403 & 27061 & 919326 & 67997 & 824267 & 892264 & 83,72 \\
\hline Murcia & 2418018 & 54891 & -158002 & 13084 & 2939772 & 17828 & 2791102 & 2808930 & 128,30 \\
\hline $\begin{array}{l}\text { C. Valen- } \\
\text { ciana }\end{array}$ & 9230129 & 1110957 & -1186155 & 1080654 & 10235586 & 308286 & 8846645 & 9154931 & 121,09 \\
\hline Aragón & 29932878 & 111680 & 249987 & 42334 & 3397290 & 81906 & 3273049 & 3354955 & 96,13 \\
\hline C. Mancha & 3528728 & 985155 & 87857 & 207366 & 4809107 & 11144 & 4590596 & 4601741 & 100,13 \\
\hline Canarias & 1773399 & 2195333 & 80492 & 202288 & 4251514 & 260839 & 3788386 & 4049226 & 113,98 \\
\hline \begin{tabular}{|l|} 
Extrema- \\
dura
\end{tabular} & 1636403 & 703548 & 391069 & 115619 & 2846640 & 7613 & 2723408 & 2731021 & 99,91 \\
\hline Baleares & 2552367 & -104134 & .586051 & 218834 & 2081015 & 108387 & 1753793 & 1862181 & 125,68 \\
\hline Madrid & 17162965 & -2654063 & .584356 & 239547 & 14164092 & 997758 & 12926787 & 13924545 & 101,13 \\
\hline C. León & 4974882 & 756546 & 395804 & 230440 & 6357673 & 14996 & 6112236 & 6127233 & 98,13 \\
\hline TOTAL & 86003404 & 7680442 & 1234902 & 4016410 & 98935160 & 5419481 & 89499268 & 94918749 & 102,99 \\
\hline
\end{tabular}

Fuente: elaboración propia a partir de Ministerio de Hacienda y Administraciones Públicas (2015). 


\section{CONCLUSIONES}

En los últimos años se ha avanzado especialmente en el campo de una asignación más equitativa y eficiente en de los recursos asignados a sanidad si la comparamos con sus inicios hace décadas en países como el nuestro (Artells, 1980). Asimismo, son continuas las mejoras para conseguir una adecuada evaluación de programas y políticas públicas en el sector sanitario (Fuchs, 2000; Zweifel, 2013; Hansen et al., 2015).

Con estas premisas, en este artículo se ha abordado el estudio del sector sanitario y su nivel de gasto sanitario dentro del gasto social de cualquier moderno Estado del Bienestar. Luego, se ha descrito la situación actual de su financiación así como su problemática y retos de futuro a fin de poner en valor la aportación fundamental de la sanidad al contexto socio-económico actual. Pasamos así a enumerar a continuación las principales conclusiones que hemos obtenido en este ejercicio:

1. Sigue existiendo cierto desequilibrio financiero vertical, pues las variables que se utilizan para determinar las necesidades de gasto regionales así como su ponderación generan amplias diferencias en financiación per cápita ajustada y la necesidad continua de tener que adaptarla a las necesidades de gasto reales. Cabe señalar que la sanidad no es una excepción en este caso.

2. Según esto, de cara a la próxima reforma del sistema de financiación, pensamos que se puede avanzar más en este campo superando así el statu quo inicial autonómico. A su vez, las variables utilizadas para identificar las necesidades de gasto en sanidad se pueden desagregar aún más intentando ajustar las amplias diferencias entre CC. AA. sin desincentivarlas fiscalmente. De esta forma, consideramos que, por ejemplo, el número de personas dependientes o afectadas por enfermedades crónicas pueden servir para aproximar, aún más, el gasto de las CC. AA. en servicios como la sanidad y servicios sociales sin olvidar su impacto en el grado de utilización de servicios (tanto en atención primaria como especializada), y en definitiva en el gasto sanitario.

3. Una mejor evaluación del gasto autonómico contribuirá a diseñar mejor sus necesidades y suficiencia financiera en sintonía con la realidad de la coyuntura económica de cada momento. Controlar bien el impacto económico sobre el sector sanitario de efectos exógenos como el envejecimiento poblacional o las cronicidades (que requieren más tratamiento durante más tiempo) exigirá nuevas soluciones en forma de fondos específicos y vincular las nuevas inversiones en el Sistema Nacional de Salud a que demuestren que realmente sean coste-efectivas. 


\section{David Cantarero Prieto}

\section{BIBLIOGRAFÍA}

ARTELLS, J. J. (1980), "La economía de la salud en perspectiva", Cuadernos de Economía, núm. 8(21), pp. 5-20.

CANTARERO, D. y OLIVA, J. (2016), "Economía de la salud: herramientas para la evaluación de programas y políticas pública", Revista de Evaluación de Programas y Políticas Públicas, núm. 6, pp. 1-8.

CONDE RUIZ, J. I., DÍAZ, M., MARÍN, C. y RUBIO-RAMÍREZ, J. (2016), "Sanidad, Educación y Protección Social: Recortes durante la crisis", Estudios sobre la Economía Española-2016/17, Observatorio Fiscal y Financiero de las CCAA.

FUCHS, V. (2000), "The future of Health Economics", Journal of Health Economics, núm. 19, pp, 141-157.

FUNDACIÓN BBVA e IVIE (Instituto Valenciano de Investigaciones Económicas) (2015), Gasto en los servicios públicos fundamentales en España y sus comunidades autónomas (2002-2013). Base de datos del informe correspondiente disponible en: http://www.fbbva.es/TLFU/tlfu/esp/areas/econosoc/bbdd/gastos_servicios_publicos_comunidades.jsp

HANSEN, F., ANELL, A., GERDTHAM, U. y LYTTKENS, C. H. (2015), "The future of health economics: The potential of behavioral and experimental economics", Nordic Journal of Health Economics, vol. 3, núm. 1, pp. 68-86.

LÓPEZ-CASASNOVAS, G. (2015), El bienestar desigual: qué queda de los derechos y beneficios sociales tras la crisis, Ediciones Península Atalaya, Barcelona.

LÓPEZ-CASASNOVAS, G. y LÓPEZ VALCARCEL, B. (2016), "El sistema sanitario en España, entre lo que no acaba de morir y lo que no termina de nacer", Papeles de Economía Española, núm. 147, pp. 190-211.

MONES, M. A., BALLETBO, A. y DE LA FUENTE, A. (2016), "Elementos para un nuevo sistema de financiación de las comunidades autónomas. Discusión y conclusiones", FEDEA Policy Papers-2016/09.

OECD, OECD Health Data 2016, París.

OECD, OECD Health at a Glance: Europe 2016, París.

PASCUAL, M. y CANTARERO, D. (2013), "Understanding health economics: a review of efficiency, equity and inequalities studies", Estudios de Economía Aplicada, vol. 31, núm. 2, pp. 1-22.

ZWEIFEL, P. (2013), "The present state of health economics: a critique and an agenda for the future", European Journal of Health Economics, núm. 14, pp. 569-571. 